\title{
Gut-derived Enterococcus faecium from ulcerative colitis patients promotes colitis in a genetically susceptible mouse host
}

\author{
Jun Seishima ${ }^{1 \dagger}$, Noriho lida ${ }^{1 \dagger}$, Kazuya Kitamura', Masahiro Yutani ${ }^{2}$, Ziyu Wang ${ }^{1}$, Akihiro Seki ${ }^{1}$, Taro Yamashita', \\ Yoshio Sakai', Masao Honda, Tatsuya Yamashita', Takashi Kagaya', Yukihiro Shirota', Yukako Fujinaga², \\ Eishiro Mizukoshi ${ }^{1 *}$ and Shuichi Kaneko ${ }^{1}$
}

\begin{abstract}
Background: Recent metagenomic analyses have revealed dysbiosis of the gut microbiota of ulcerative colitis (UC) patients. However, the impacts of this dysbiosis are not fully understood, particularly at the strain level.

Results: We perform whole-genome shotgun sequencing of fecal DNA extracts from 13 healthy donors and 16 UC and 8 Crohn's disease (CD) patients. The microbiota of UC and CD patients is taxonomically and functionally divergent from that of healthy donors, with E. faecium being the most differentially abundant species between the two microbial communities. Transplantation of feces from UC or CD patients into $1 / 10^{-/-}$mice promotes pathological inflammation and cytokine expression in the mouse colon, although distinct cytokine expression profiles are observed between UC and CD. Unlike isolates derived from healthy donors, E. faecium isolates from the feces of UC patients, along with E. faecium strain ATCC 19434, promotes colitis and colonic cytokine expression. Inflammatory E. faecium strains, including ATCC 19434 and a UC-derived strain, cluster separately from commercially available probiotic strains based on whole-genome shotgun sequencing analysis. The presence of $E$. faecium in fecal samples is associated with large disease extent and the need for multiple medications in UC patients.
\end{abstract}

Conclusions: E. faecium strains derived from UC patients display an inflammatory genotype that causes colitis.

Keywords: Inflammatory bowel disease, Crohn's disease, Microbiota, Metagenome

\section{Background}

The hundreds of trillions of microbes in the gastrointestinal tract contribute to many host physiological processes including nutrient acquisition and development of the gut immune system, while dysbiosis of the microbiota can contribute to the development of several diseases [1]. The commensal gut microbiota is closely related to the pathogenesis of inflammatory bowel diseases (IBD), including ulcerative colitis (UC) and Crohn's disease (CD) [2]. A significant amount of information on the role of the gut microbiota in IBD patients has been accumulated based on clinical studies, metagenome analyses, and animal experiments. The recent

\footnotetext{
* Correspondence: eishirom@m-kanazawa.jp

† Jun Seishima and Noriho lida contributed equally to this work.

1 Department of Gastroenterology, Graduate School of Medical Sciences,

Kanazawa University, Kanazawa, Ishikawa 920-8641, Japan

Full list of author information is available at the end of the article
}

development of high-throughput metagenome sequencing techniques and analysis methods has uncovered dysbiosis of the microbiota of IBD patients, with a decrease in microbial diversity compared with healthy individuals [3-5]. In addition, the gut community of IBD patients shows an increased prevalence of the phylum Proteobacteria [6], including inflammatory species Escherichia coli [4], and a decrease in the phylum Firmicutes [6], including anti-inflammatory species Faecalibacterium prausnitzii $[4,7]$. While metagenomic analysis can reveal an association between dysbiosis and disease, animal studies can demonstrate a causative association between specific bacteria and the pathogenesis of colitis. According to previous mouse studies, F. prausnitzii [8], Bacteroides fragilis [9], and Clostridia species [10] attenuate intestinal inflammation via various mechanisms, including induction of IL-10 and regulatory $\mathrm{T}$ cells in the colon, whereas

(C) The Author(s). 2019 Open Access This article is distributed under the terms of the Creative Commons Attribution 4.0 International License (http://creativecommons.org/licenses/by/4.0/), which permits unrestricted use, distribution, and 
Escherichia coli [11] and Enterococcus faecalis [11, 12] promote colitis in mouse models.

Although previous studies have built up a significant body of information, questions remain about the causal relationship between human IBD pathogenesis and microbiota dysbiosis. Firstly, because of the complexity of the gut microbiota and the multifactorial nature of IBD, it is unclear exactly which bacterial species in the dysbiotic IBD microbiota are responsible for the pathogenesis of colitis. Because few reports reproduce the microbial composition of the dysbiotic microbiota in colitic mouse models, it is unclear whether decreases or increases in a single antiinflammatory or pro-inflammatory species are truly responsible for IBD pathogenesis. Secondly, the specific genotypes of bacterial species putatively responsible for IBD pathogenesis are yet to be elucidated. Metagenomic analysis of the gut microbiota, particularly $16 \mathrm{~S}$ ribosomal RNA (rRNA) sequencing-based approaches, cannot identify the composition of the microbiota at the strain level. Therefore, it is difficult to judge based only on metagenomic analysis whether the decreased or increased prevalence of a species in the IBD microbiota has a pathogenic effect.

In the present study, to answer these questions, we attempted to find a link between human metagenome data and phenotype in a mouse model of colitis. We first analyzed whole-genome shotgun sequencing data corresponding to fecal metagenomes obtained from patients with IBD. Feces from the same subjects was then transplanted into $I l 10^{-/-}$mice to assess the causal relationship between dysbiosis of the microbiota and intestinal inflammation. Finally, the genotype of a bacterial species possibly responsible for inflammation, Enterococcus faecium, was identified by sequencing multiple strains isolated from UC patients. Thus, we clearly showed a causal relationship between UC-derived bacterial strains and colonic inflammation.

\section{Results}

The gut microbiota of IBD patients is taxonomically and functionally divergent from that of healthy donors

To investigate taxonomic and functional differences in gut microbiota between patients with IBD and healthy controls, fecal samples were collected from 13 healthy donors (HD), 16 UC patients, and $8 \mathrm{CD}$ patients. The $\mathrm{CD}$ patients were younger and had higher serum Creactive protein levels than the $\mathrm{HD}$, although no difference was observed between HD and UC patients in other baseline characteristics (Additional file 1: Table S1). The extent of disease in UC patients was either leftsided colitis or pancolitis (left-sided colitis, 68.8\%; pancolitis, 31.3\%; Additional file 1: Table S2). Fifteen of the 16 UC patients were being treated with medications, including mesalazine, corticosteroids, tacrolimus, azathioprine, mercaptopurine, and TNF antagonist, and both active and inactive colitis patients were represented in the UC cohort according to total Mayo scores (average Mayo score, 3.25; Additional file 1: Table S2). All the $\mathrm{CD}$ patients were being treated with medications, including mesalazine, corticosteroids, azathioprine, and TNF antagonist, and in most cases, the disease was inactive based on the Crohn's disease activity index (average CDAI, 71.1; Additional file 1: Table S3).

Whole-genome shotgun sequencing of fecal DNA samples revealed significant differences in abundance of 20 and 18 bacterial species between the HD and UC, and HD and CD communities, respectively (Fig. 1a, b). Of these species, E. faecium and Escherichia coli had the highest linear discriminative analysis (LDA) scores in the $\mathrm{UC}$ and $\mathrm{CD}$ samples, respectively, compared with the HD samples (Fig. 1a, b). Anti-inflammatory species $F$. prausnitzii was less abundant in the UC communities compared with the HD communities in this analysis (Fig. 1a), as previously reported [7]. Seven species, including Collinsella aerofaciens, Ruminococcus obeum, Dorea longicatena, Eubacterium hallii, Coprococcus comes, Adlercreutzia equolifaciens, and a Lachnospiraceae sp. bacterium, were less abundant in both the UC and $\mathrm{CD}$ communities compared with the HD community (Fig. 1a, b). Metabolic pathway analysis of the gut microbiota revealed that 38 and 35 Kyoto Encyclopedia of Genes and Genomes (KEGG) pathways were significantly different between the HD and UC, or HD and CD samples, respectively (Additional file 1: Tables S4 and S5). Pathways involved in glycerophospholipid metabolism were less abundant in both the $\mathrm{UC}$ and $\mathrm{CD}$ microbiota compared with those of the HD patients (Additional file 1: Tables S4 and S5). Analysis of the UC microbiota showed that pathways involved in DNA replication and repair, including base excision repair and mismatch repair, were more abundant compared with the microbiota of the HD (Additional file 1: Table S4). In $\beta$-diversity analysis and principal coordinate analysis (PCoA), the UC and CD microbiota showed taxonomically (Fig. 1c) and functionally (Fig. 1d) differential plots compared with the HD microbiota $(P=0.001$, PERMANOVA). Because the abundance of E. faecium in UC patients has not previously been reported, the difference in abundance of fecal E. faecium between HD and UC patients was confirmed by quantitative PCR, which is more sensitive in detection than metagenomic sequencing analysis (Fig. 1e). Thus, these results confirm both taxonomic and functional differences in the gut microbiota of UC and CD patients compared with the HD.

\section{The gut microbiota of UC and CD patients cause colitis in $1110^{-1-}$ mice}

To explore a causal relationship between the gut microbiota and colitis, the fecal samples used in the 

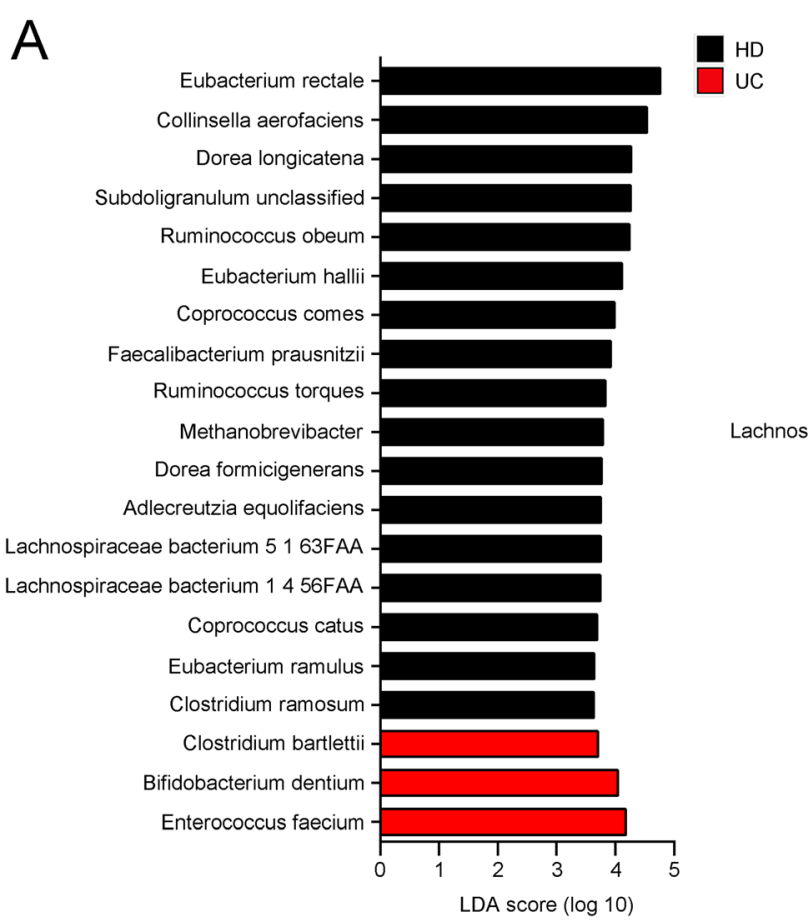

B

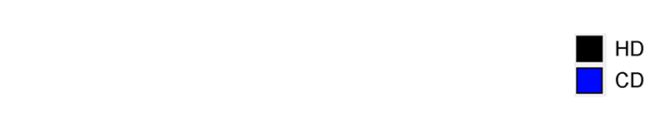

C

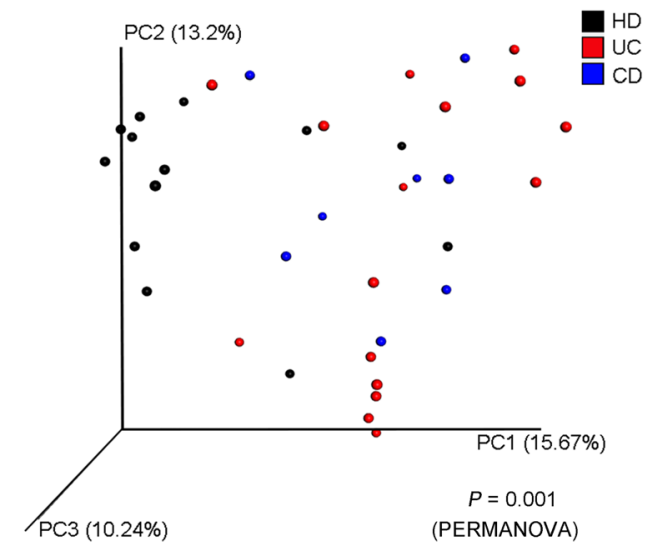

D
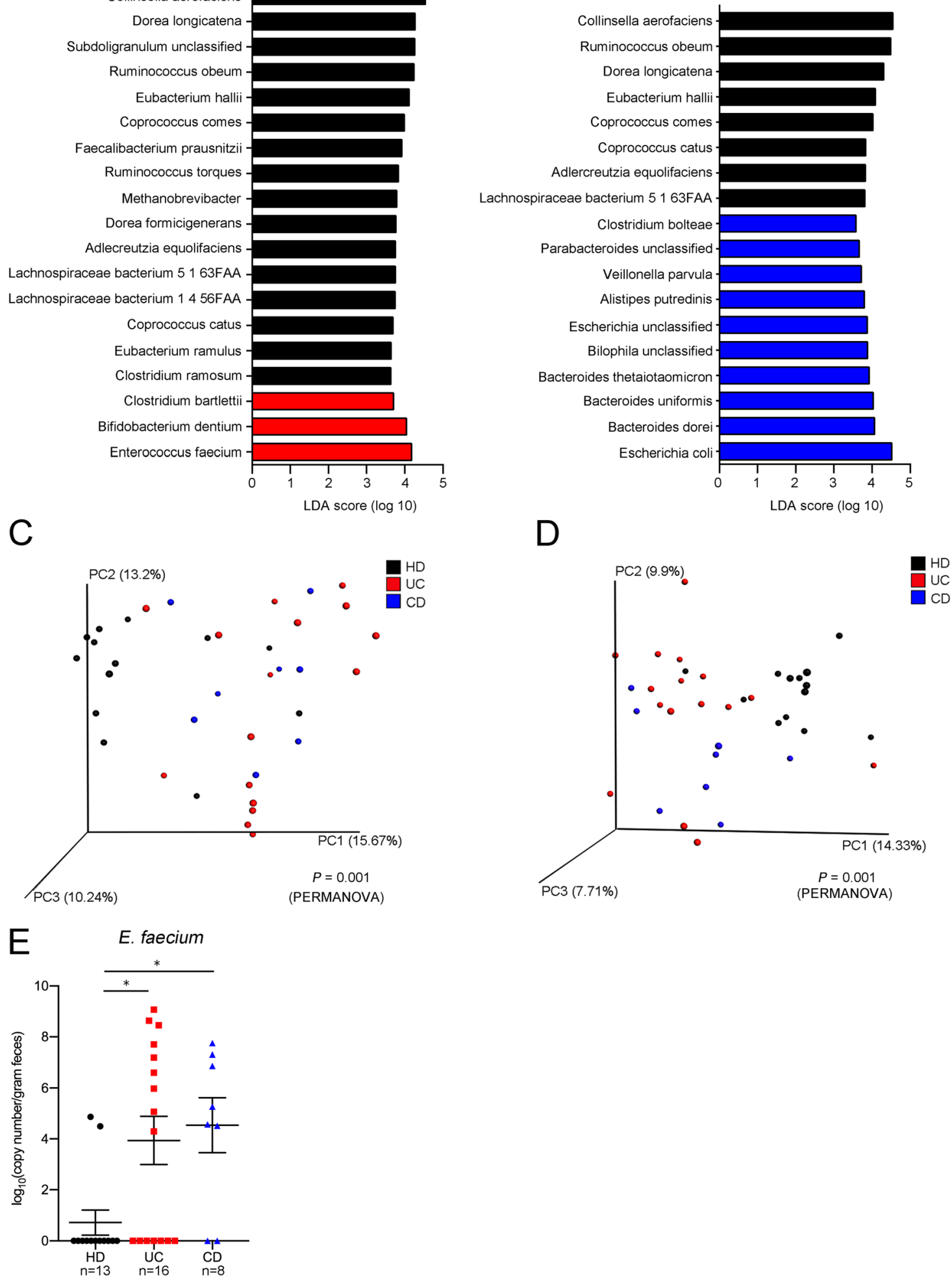

Fig. 1 (See legend on next page.) 
(See figure on previous page.)

Fig. 1 The microbiota of inflammatory bowel disease (IBD) patients is taxonomically and functionally divergent from that of healthy donor (HD) subjects. a Linear discriminative analysis (LDA) was performed using linear discriminative analysis effect size (LEfSe) to identify significant differences in relative abundance of various taxonomic groups in the feces of HD subjects compared with ulcerative colitis (UC) patients, or $\mathbf{b}$ HD patients compared with Crohn's disease (CD) patients. Differentially abundant genera for which the corresponding LDA scores indicate $P<0.05$ are shown in the graphs. c Distances between the patient groups were calculated using the Bray-Curtis index based on taxonomic data or $\mathbf{d}$ Kyoto Encyclopedia of Genes and Genomes (KEGG) pathway data and visualized by principal coordinate analysis. Significant differences between groups were determined by PERMANOVA, and $P$ values are shown at the bottom of the plots. e Copy number of Enterococcus faecium in feces was determined by PCR. ${ }^{*} P<0.05$

sequencing analysis were transplanted into $1 l 10^{-/-}$ colitis-susceptible mice. Fecal microbiota samples from each of the subjects were transplanted into at least two microbiota-depleted $I l 10^{-1-}$ mice, and colonic inflammation was evaluated on day 28 post-transplantation by pathology and gene expression analyses. Although longitudinal analysis of the fecal microbiota following fecal transplantation revealed that the composition of the human gut community was not completely restored in the transplanted mice (Additional file 2: Figure S1a and b), the composition of the murine microbiota was uniquely dependent on the human subjects' microbiota and remained stably segregated from the composition in other mice up to 28 days post-transplantation (Additional file 2: Figure S1c, $P=0.001$, PERMANOVA). Effects of cage difference (Additional file 2: Figure S1d) and repeats of transplantation (Additional file 2: Figure S1e) were modest, and PCoA plots were significantly differentially segregated by differences between the donor feces (Additional file 2: Figure S1d, $P=0.001$; Additional file 2: Figure S1e, $P=0.029$ : PERMANOVA). Therefore, we evaluated the effects of these unique human-derived microbial communities on the severity of colitis in the mouse model.

Body weight of mice transplanted with the UC or the CD patient microbiota increased less than that of mice transplanted with the HD microbiota or the microbiotadepleted controls (Fig. 2a, b). Pathological changes were more severe in rectum segments than in the proximal colon in this mouse model (Additional file 2: Figure S2). A greater number of mice transplanted with the UC or CD microbiota showed epithelial hyperplasia with lymphoplasmacytosis, obliteration of normal architecture, and erosion, particularly in the rectal segments, compared with the HD group and microbiota-depleted controls (Fig. 2c and Additional file 2: Figure S2). The average pathology score for the colon was significantly higher for the UC and CD microbiota-transplanted mice compared with the HD microbiota-transplanted mice (Fig. 2d, e). In addition, body weight change was negatively correlated with pathology score (Fig. 2f), implying that inflammation in the colon was responsible for the decreased body weight.

Because expression of the inflammatory cytokines Tnf and $I l 1 b$ in colon tissues was significantly higher in mice transplanted with $\mathrm{HD}, \mathrm{UC}$, and $\mathrm{CD}$ microbiota than in microbiota-depleted control mice (Fig. 3a), those cytokines were considered to be induced in response to various bacterial species present in the HD feces as well as the IBD feces. Among the three groups, the UC microbiota induced the highest levels of expression of Tnf and Il1b (Fig. 3a). The relative expression of Tnf in the colon was positively correlated with pathology scores in the same tissue (Fig. 3b). In comparison, the expression of Il6 and $I l 17 a$ was significantly higher in the colon tissues of UC microbiota-transplanted mice than in the HD or CD microbiota-transplanted mice or in the microbiotadepleted controls. However, the colonic expression of Il23a was significantly higher in the CD microbiotatransplanted mice than in the HD or UC microbiotatransplanted mice or the microbiota-depleted controls (Fig. 3a). The pattern of cytokine expression varied in individual mice, dependent on the feces of the individual human donor (Additional file 2: Figure S3). Therefore, the gene expression profiles of cytokines in the colon appear to be dependent on the fecal microbiota of the subjects.

\section{The abundance of Enterococcus in the fecal microbiota of} mice is associated with inflammation in the colon

Because the gut community composition of each of the human subjects was not completely reproduced in the transplanted mice, the characteristics of the gut microbiota of the transplanted mice were next examined by $16 \mathrm{~S}$ rRNA-based metagenomic analysis. Significant differences in the abundance of nine genera were observed between the HD and the UC microbiota-transplanted mice, whereas only three genera were significantly altered between the HD and CD microbiota-treated mice (Fig. 4a). Enterococcus and Enterobacter were more abundant in the UC microbiota-transplanted gut communities compared with the HD group, while Ruminococcus and Anaerotruncus were less abundant in the UC group than in the HD microbiota-transplanted mice. Paraprevotella and Enterobacter were significantly more abundant in the CD microbiota-transplanted mice compared with the HD group. Because E. faecium was enriched in the microbiota of human UC patients compared with HD subjects (Fig. 1a), colonization of E. faecium in mouse colon was examined by culture of murine feces. On day 28 after fecal transplantation, 


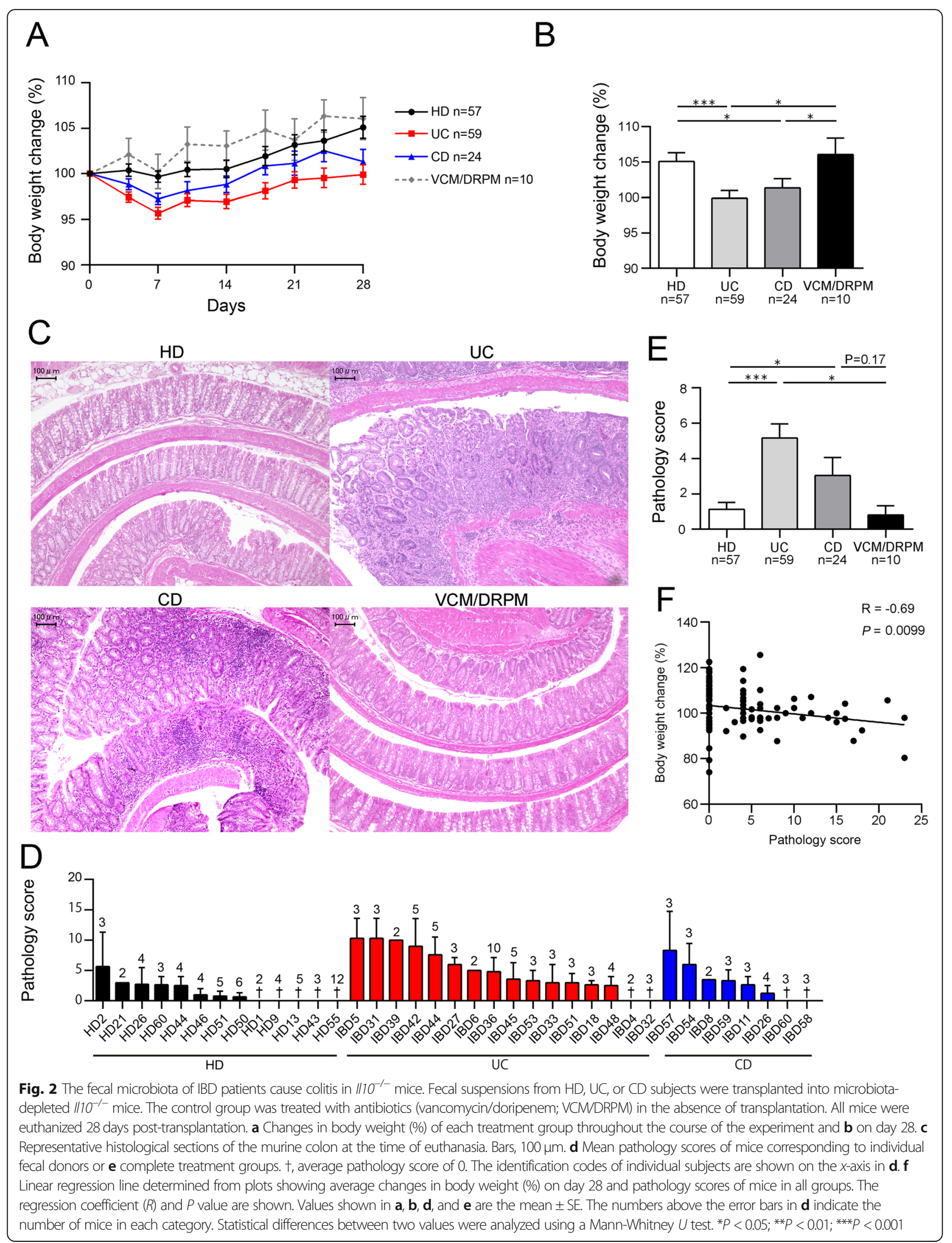




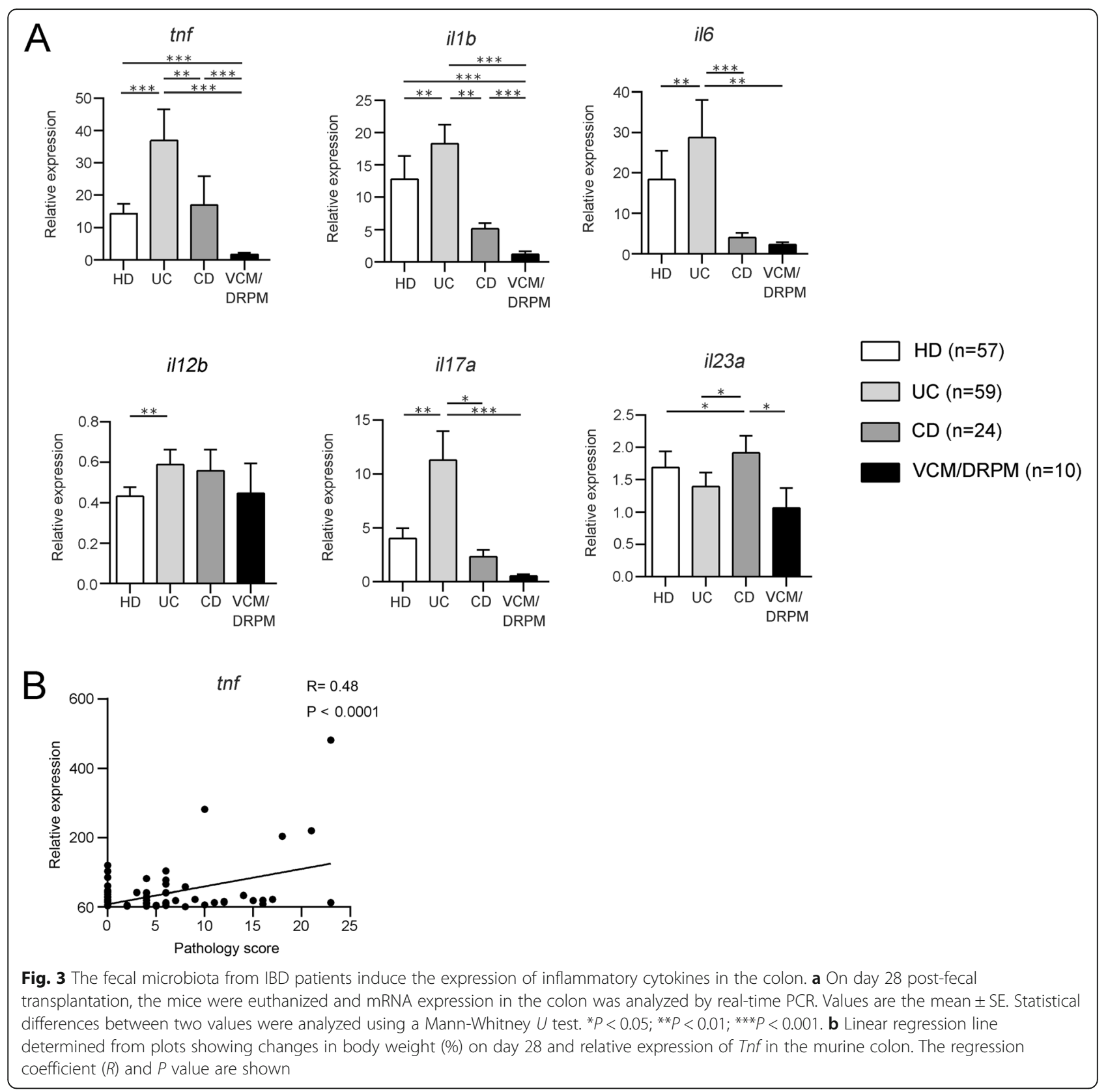

colonization by E. faecium was detected significantly more in UC microbiota-transplanted mice than in HD microbiota-transplanted mice. No E. faecium was detected in any group before fecal transplantation (Additional file 2: Figure S4). Pathology and cytokine expression data from Figs. 2 and 3 were reanalyzed with respect to the proportion of Enterococcus in the microbiota of the mice determined by $16 \mathrm{~S}$ rRNA-based metagenomic sequencing. Pathology scores and expression of Tnf, Illb, Il6, and $I l 17 a$ in the colon in mice positively correlated with the proportion of Enterococcus (Fig. 4b). However, when the copy number of $E$. faecium in mouse feces was determined by sensitive detection by quantitative PCR
(Additional file 2: Figure S5a), the number of E. faecium only correlated with $I l 17 a$ expression (Fig. 4b and Additional file 1: Table S6). Because E. faecalis is another major species that often colonizes clinical patients, the copy number of $E$. faecalis in mouse feces after fecal transplantation was also determined by PCR (Additional file 2: Figure S5b). Although the number of E. faecalis alone only correlated with $\mathrm{Ill7a}$ expression, the sum of the number of E. faecium and $E$. faecalis positively correlated with pathology scores and expression of $I l 1 b, I l 6, I l 12 b$, and $I l 17 a$ (Fig. 4b and Additional file 1: Table S6). Thus, the presence of the genus Enterococcus, including E. faecium and 


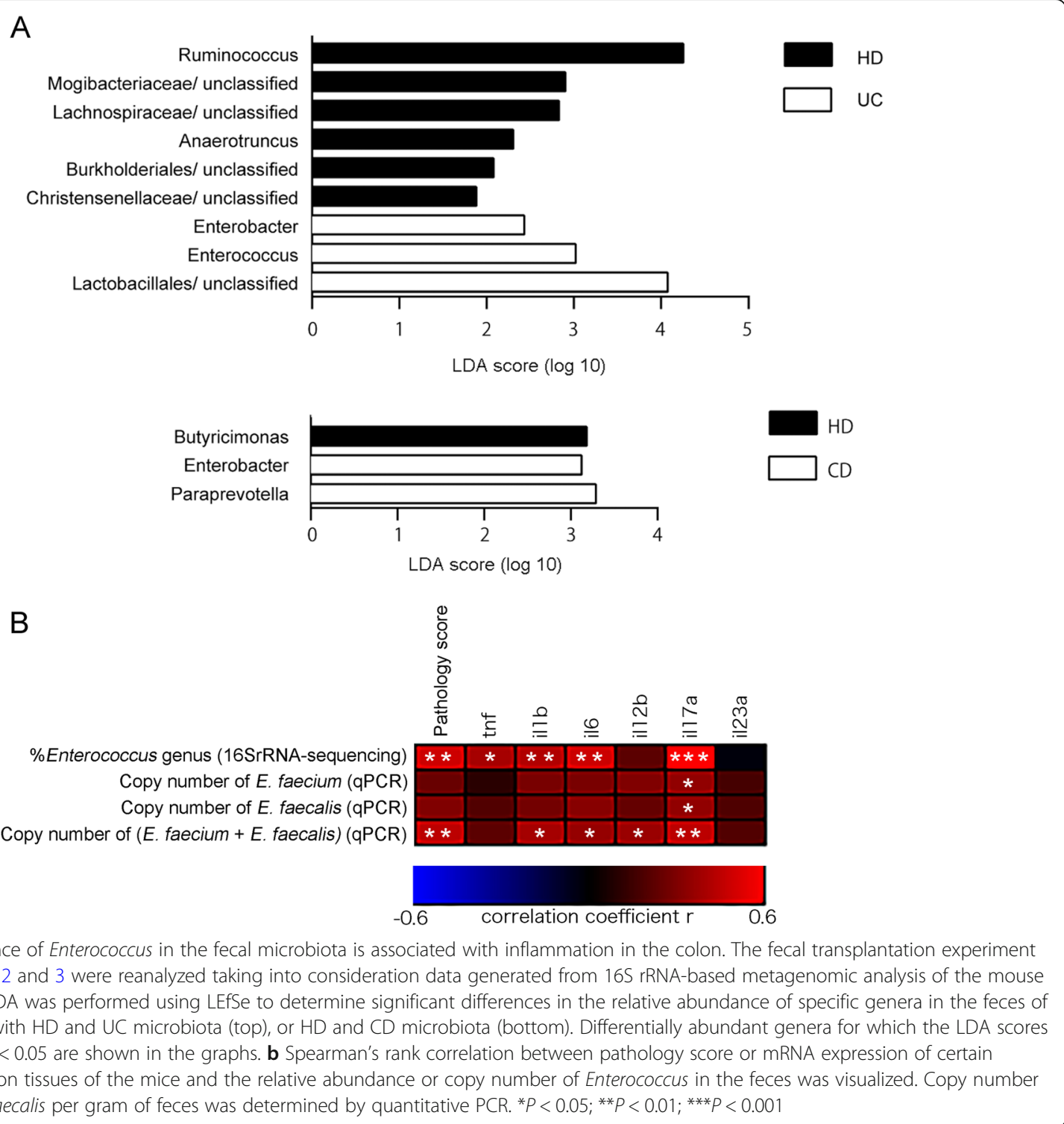

E. faecalis, seemed to be correlated with colitis in mice after fecal transplantation.

\section{E. faecium causes colitis in $1110^{-/-}$mice}

E. faecalis causes colitis in $I l 10^{-1-}$ mice according to a previous report [13], but a remaining question was whether E. faecium is causally involved in colitis. To further investigate the inflammatory effects of E. faecium in the colon, $E$. faecium strain ATCC 19434 was orally and rectally inoculated into the gut of microbiota-depleted $\mathrm{IllO}^{-/-}$mice and colonization was confirmed (Additional file 2: Figure S6a and b). Fecal microbiota from selected subjects, HD subject 55 and UC patient IBD36 as negative and positive controls for inflammation, respectively, were also separately transplanted into microbiota-depleted mice. Increases in body weight were slower in the mice colonized with ATCC
19434 or IBD36 microbiota compared with mice transplanted with HD55 microbiota or the microbiota-depleted controls (Fig. 5a, b). Colon tissues of mice inoculated with ATCC 19434 or the IBD36 microbiota showed epithelial hyperplasia with lymphoplasmacytosis, obliteration of normal architecture, and erosion, particularly in the rectal segments, and mice belonging to these two groups had higher pathology scores compared with mice inoculated with HD55 microbiota and the microbiota-depleted controls (Fig. 5c, d). ATCC 19434 colonization significantly increased the expression of Tnf, Illb, Il6, Il17a, and Il12b in colon tissues compared with HD55 microbiota transplantation. Therefore, we concluded that E. faecium ATCC 19434 is an inflammatory strain capable of promoting the expression of inflammatory cytokines and causing pathological changes in colon tissues. 


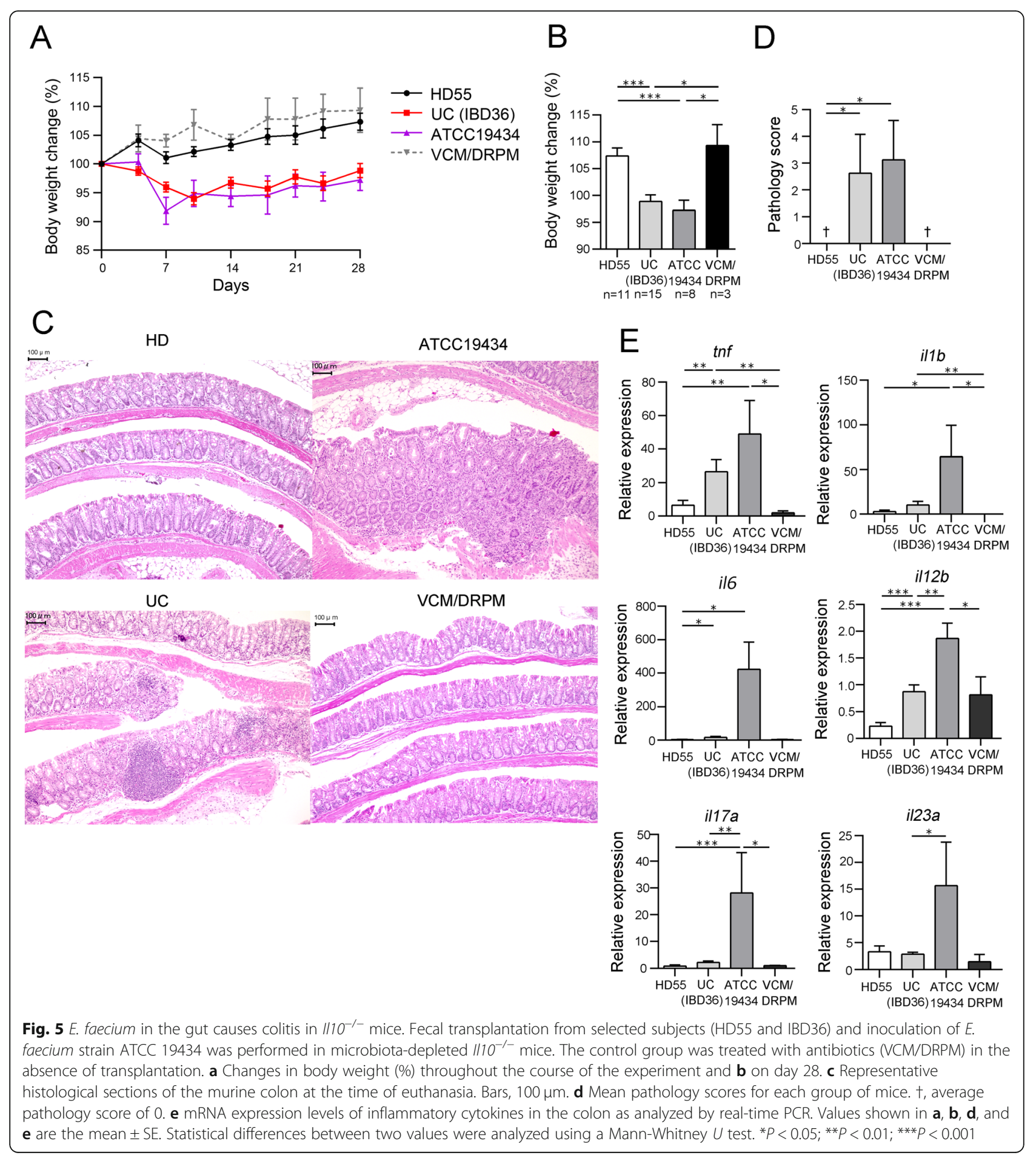

Subject-derived $E$. faecium strains induce different pathological changes and cytokine expression profiles in the colon

To examine whether E. faecium strains isolated from different subjects could promote colitis, multiple strains were isolated from the fecal samples. Colonization of strains in mice was confirmed by culture (Additional file 2: Figure
S6c to f). Increases in the body weight of $1 l 10^{-1-}$ mice inoculated with the UC microbiota-derived E. faecium strains IB18a or IB51a were slower than those recorded for HD55 microbiota-transplanted mice (Fig. 6a, b). In accord with body weight change, strain HD26a only caused modest pathological changes in the colon, whereas inoculation of IB18a or IB51a resulted in higher pathology 


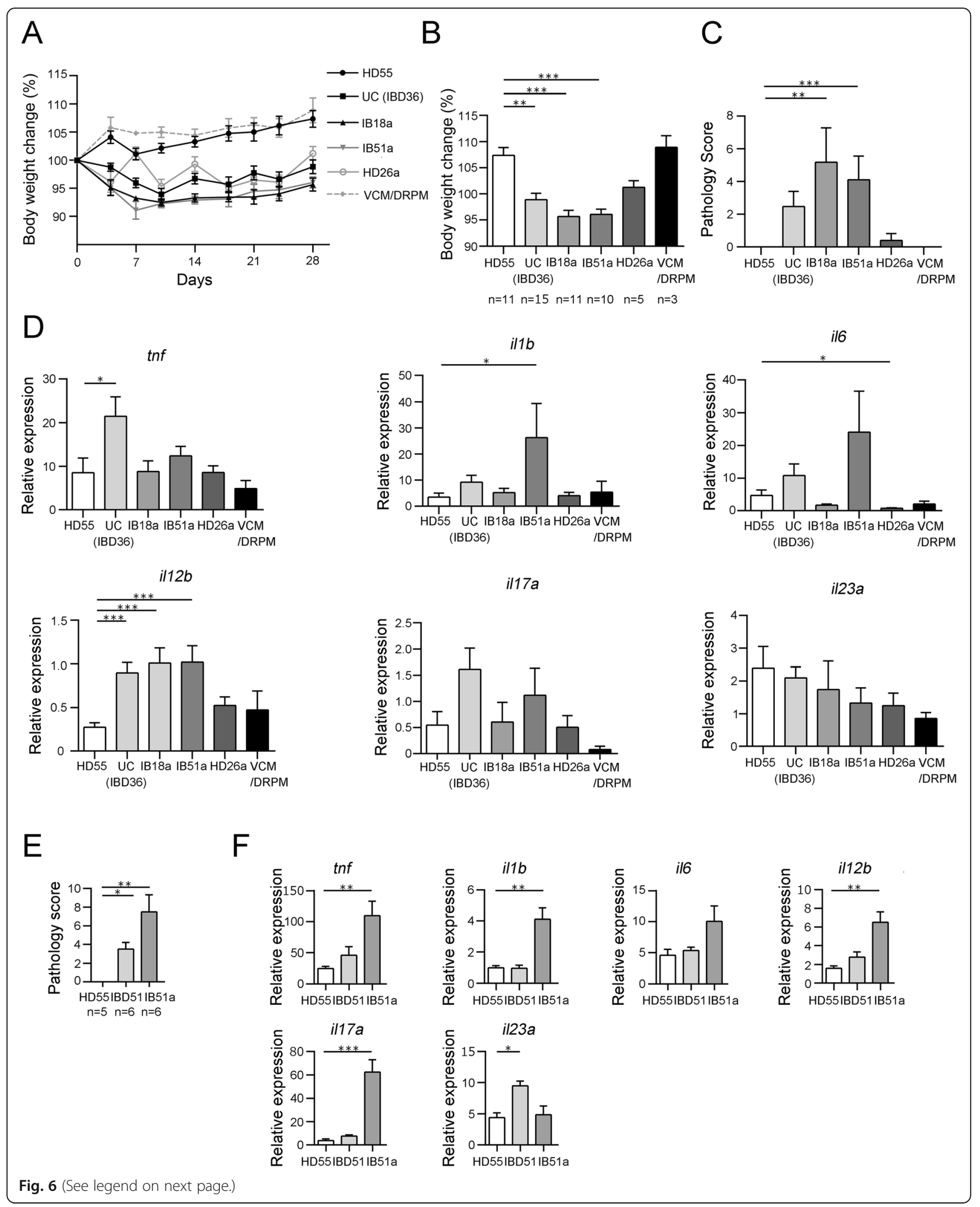


(See figure on previous page.)

Fig. 6 Subject-derived E. faecium strains lead to different pathology and cytokine expression profiles in the colon. Fecal suspensions from selected subjects (HD55 and IBD36) and E. faecium strain IB18a, IB51a, or HD26a suspensions were transplanted or inoculated into microbiotadepleted $/ / 10^{-/-}$mice. The control group was treated with antibiotics (VCM/DRPM) in the absence of transplantation. a Changes in body weight (\%) throughout the course of the experiment and $\mathbf{b}$ on day 28. c Mean pathology scores of mice from each treatment group. $\mathbf{d}$ mRNA expression levels of inflammatory cytokines in the colon as analyzed by real-time PCR. e, f Suspension of HD55 or IBD51 feces or strain IB51a was gavaged into germ-free $/ 110^{-1-}$ mice. e Mean pathology scores of mice from each treatment group. $\mathbf{f}$ mRNA expression levels of inflammatory cytokines in the colon as analyzed by real-time PCR. Values shown in a-f are the mean \pm SE. Statistical differences between a value and the HD55 control were analyzed using the Kruskal-Wallis test followed by Dunn's test. ${ }^{*} P<0.05$; ${ }^{*} P<0.01$; ${ }^{* *} P<0.001$

scores in the colon compared with HD55 microbiota transplantation (Fig. 6c).

Consistent with these findings, strain HD26a did not increase the expression of inflammatory cytokines in colon tissues compared with HD55 microbiota transplantation (Fig. 6d). Interestingly, colon tissue cytokine expression profiles differed following inoculation with IB18a or IB51a. IB51a induced higher levels of expression of $I l 1 b$ and $I l 12 b$ compared with the HD55 microbiota, while IB18a only induced higher expression of $I l 12 b$ (Fig. 6d). Commercially available E. faecium strain SF68, which is used as a probiotic drug for animals [14], was also tested by inoculation into microbiota-depleted $I l 10^{-/-}$mice. SF68 did not cause pathological inflammation and increases of colonic cytokine expression, whereas UC-derived strain IB44a caused pathological colitis and increased expression of Thf, $I l 12 b$, and Il17a (Additional file 2: Figure S7). Next, UC-derived strain IB51a was inoculated into germ-free $\mathrm{Il10}^{-/-}$mice to see whether a single strain was sufficient to induce colitis. Inoculation with IB51a induced colonization with 100 times more E. faecium than transplantation of IBD51 feces that was the origin of IB51a (Additional file 2: Figure S6 g). Both monocolonization with IB51a and transplantation of IBD51 feces into germ-free mice induced more severe colitis than transplantation of HD55 feces (Fig. 6e). Strain IB51a increased expression of Tnf, Il1b, Il12b, and Il17a in the colon tissue, but IBD51 feces only increased expression of Il23a compared with HD55 feces (Fig. 6f). The cellular source of IL-17 in colonic lamina propria was both Th17 cells and CD3-negative cells (Additional file 2: Figure S8a and b). TNF and IL-6 were mainly produced by CD11c ${ }^{-} \mathrm{MHC}-$ class- $\mathrm{II}^{+}$cells as well as smaller proportion of CD11 $\mathrm{c}^{+} \mathrm{MHC}-$ class- $\mathrm{II}^{+}$cells (Additional file 2: Figure S8c to f).

To assess whether the different pathology and cytokine expression profiles in the colon tissues were caused by differences in the genotypes of the various E. faecium strains, genomic DNA from each of the strains was sequenced. In total, 1683 genes (not counting genes coding for hypothetical proteins) were annotated from the DNA sequence reads derived from 10 E. faecium strains. Hierarchical clustering analysis of the 1683 genes generated 2 apparent clusters among the 10 strains (see Fig. 7a and the gene list described in Additional file 3: Figure S9). E. faecium strains NCIMB 11181 and SF68, both of which have previously been used as probiotics in animals $[14,15]$, were closely clustered, but pro-inflammatory $E$. faecium strain ATCC 19434 was in the other cluster. Three strains derived from the feces of UC patients, IB51a, IB6a, and IB44a, were clustered with proinflammatory strain ATCC 19434, while two strains derived from the feces of HD subjects, HD26a and HD50a, were clustered with probiotic strains NCIMB 11181 and SF68, although HD-derived strain HD59a was grouped into the other cluster. IB18a, which induced a different cytokine expression profile from that of strain IB51a, was distant in the plot from IB51a and was much closer to the probiotic strains (Fig. 7a and Additional file 3: Figure S9). KEGG-based analysis was used to identify metabolic pathways present in the probiotic cluster and the inflammatory cluster. The E. faecium strains in the inflammatory cluster possessed more or less abundant pathways of secondary bile acid biosynthesis or lipoic acid metabolism, respectively, compared with those in the probiotic cluster (Fig. 7b). Activity of bile salt hydrolase (BSH) which is involved in the first process of secondary bile acid biosynthesis was dependent on type of strains, and HD26a and HD50a, two strains derived from $\mathrm{HD}$ feces, lacked the $\mathrm{BSH}$ activity (Additional file 4: Figure S10a). Because lipoic acids are anti-oxidants according to previous reports [16], reactive oxygen species (ROS) level in E. faecium culture was also measured. Relative ROS level in culture supernatant of the strains in the inflammatory cluster was higher than the probiotic cluster (Additional file 4: Figure S10b and c). Taken together, these results suggest that the E. faecium strains derived from the subjects' microbiota were genetically diverse and that this genetic diversity might be associated with the pathological diversity of the strains in the colon.

\section{Presence of $E$. faecium in the gut is associated with disease extent and the requirement for combination therapy in UC patients}

To investigate the clinical association between E. faecium colonization and UC, data from the 16 UC subjects were examined in more detail. Disease characteristics and treatment regimens were obtained from medical records and were assessed to identify any association with the presence or 


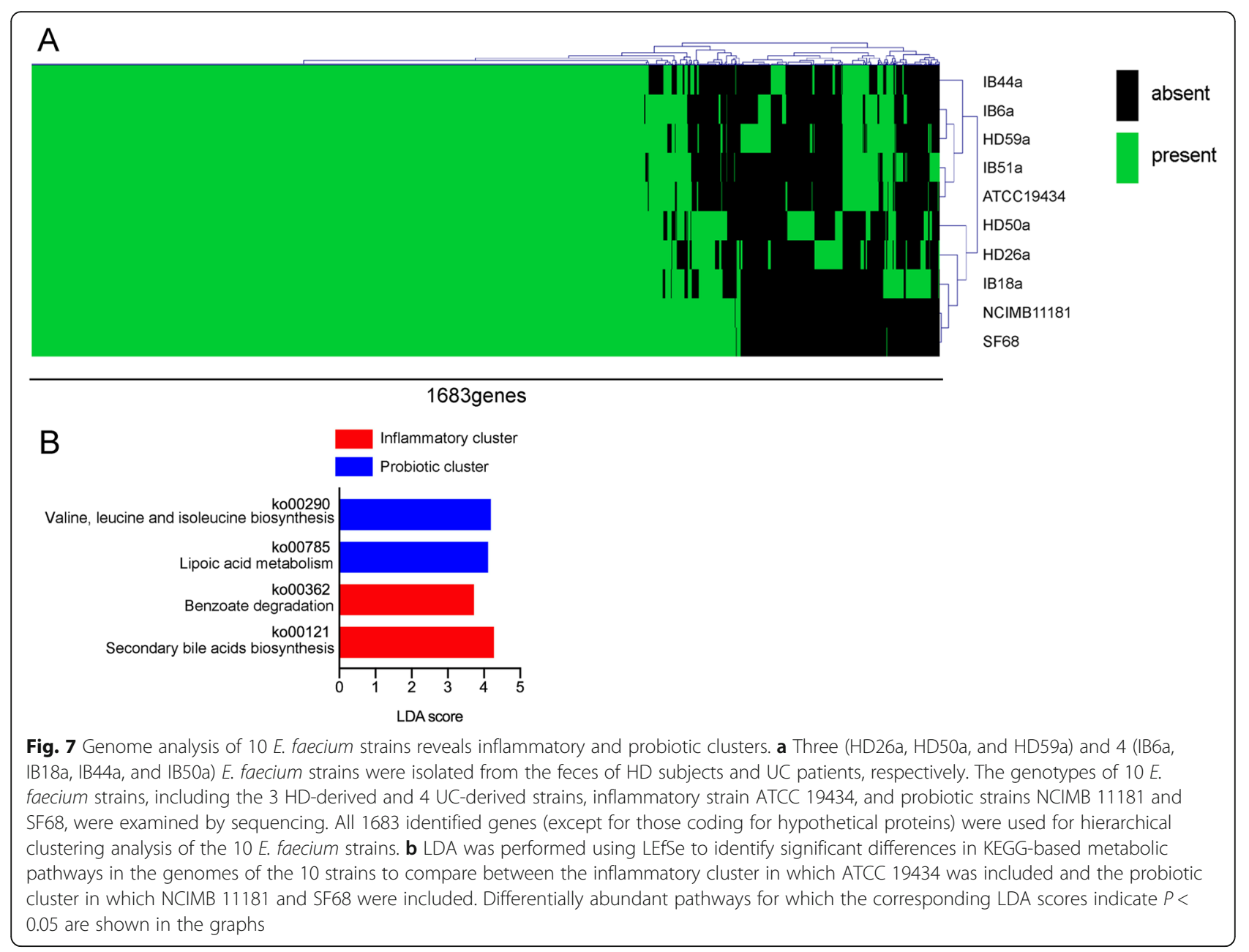

absence of E. faecium as determined from the PCR data. Fifteen UC patients received medication; the Mayo score, a disease activity score of UC, can be influenced by treatment. Thus, the presence of E. faecium was not associated with the Mayo score (Additional file 4: Figure S11). However, in terms of disease type, the prevalence of pancolitis was higher among UC patients colonized with E. faecium compared with those that were not colonized with $E$. faecium (pancolitis: E. faecium-negative, $0 \%$; E. faecium-positive, $55.6 \% ; P=$ 0.0087; Fig. 8a). In addition, E. faecium-positive UC patients tended to be treated with a greater number of mediations than UC patients without $E$. faecium in the colon (number of medications $=0$ or 1 , and 2 or 3: E. faecium-negative, 57.1\% and 42.8\%; E. faecium-positive, $11.1 \%$ and $88.8 \%$; $P=$ 0.048; Fig. 8b). Thus, the presence of E. faecium in the gut was associated with disease extent and the requirement for combination therapy in UC patients.

\section{Discussion}

A causal link between specific bacterial species and the pathogenesis of IBD remains controversial because of the multifactorial causes of IBD and difficulties surrounding reproducing the disease in mouse models. In this study, we attempted to identify a causal relationship between specific bacterial species and IBD by conducting a metagenomic analysis of colitis-susceptible $I l 10^{-/-}$mice transplanted with fecal microbiota from 37 human subjects. The fecal microbiota from both UC and CD patients caused pathological inflammation in the colon tissues of experimental animals, while microbiota from HD rarely caused severe colitis (Fig. 2c-e). The microbiota from UC patients induced expression of the Il6-Ill7a axis in the colon, whereas the microbiota from $\mathrm{CD}$ patients induced expression of Il23a (Fig. 3a). Enterococcus was differentially abundant in the microbiota of UC patients compared with the HD group (Fig. 1a), which was replicated in the mice transplanted with the UC microbiota (Fig. 4a and Additional file 2: Figure S5). E. faecium strain ATCC 19434 (Fig. 5c-e), along with strains isolated from UC patients (Fig. 6c, d), caused pathological inflammation and upregulation of cytokine expression in the colon. The genotypes of 10 analyzed $E$. faecium strains were different and could be separated into two major clusters: one containing two probiotic strains and the other containing 

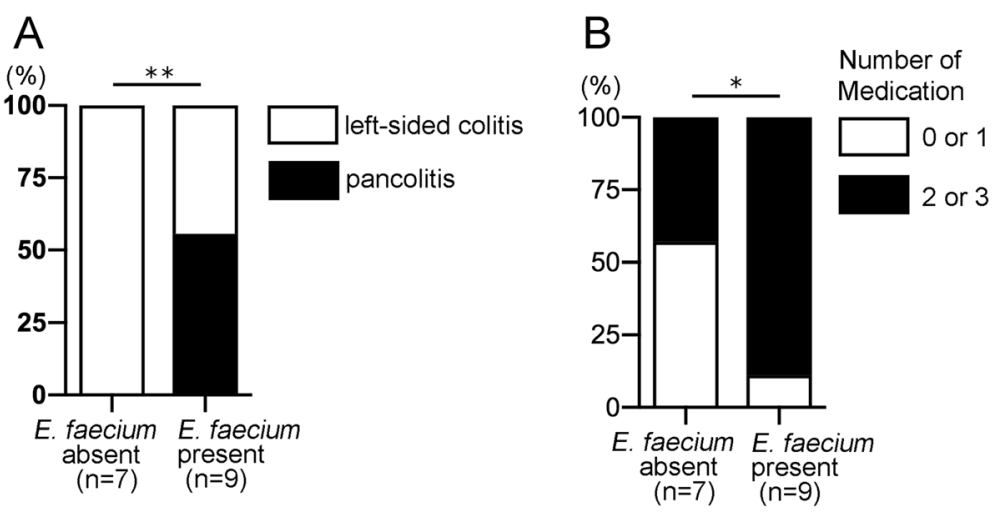

Fig. 8 The presence of E. faecium is associated with disease extent and the requirement for combination therapy. The disease characteristics and treatment regimens of the $16 \mathrm{UC}$ patients enrolled in this study were obtained from medical records and assessed to identify any association with the presence or absence of E. faecium in the gut microbiota as determined by PCR. a Proportions of UC patients with pancolitis or left-sided colitis are shown relative to the presence of $E$. faecium in the feces. $\mathbf{b}$ Proportions of UC patients treated with single or multiple mediations are shown relative to the presence of $E$. faecium in the feces. Medications included mesalazine, corticosteroids, azathioprine, mercaptopurine, tacrolimus, infliximab, and adalimumab. The number of subjects per category $(n)$ is indicated. ${ }^{*} P<0.05$ by chi-squared test

pro-inflammatory strain ATCC 19434 (Fig. 7a). Thus, these findings implicate E. faecium strains with a particular genotype in colonic inflammation in genetically susceptible hosts.

The metagenomic analysis carried out in this study revealed obvious differences in the composition of the microbiota from UC or CD patients compared with that from HD (Fig. 1c). Several of the observed differences between the IBD patients and HD were consistent with previous reports, such as the decreased abundance of $F$. prausnitzii $[7,17]$ and Eubacterium rectale [17] in the microbiota of UC patients, and the increased abundance of Escherichia species, including Escherichia coli [3], in the $\mathrm{CD}$ microbiota. According to previous reports, enterococci are more abundant in the fecal matter $[5,18]$ and mucosa [19] of CD patients compared with healthy controls. In this analysis, E. faecium was more abundant in the UC microbiota, but not the CD microbiota, compared with HD (Fig. 1a). Although the results of metagenomic analyses are often affected by large interindividual differences arising from differences in factors such as geographic location, diet, and antibiotic use, the overall results of the compositional difference between HD and IBD in the current study are fairly consistent with previous reports. Because most of the patients enrolled in this study were being treated for their disorder, it is unclear whether the observed changes in microbiota are associated with the disease onset. Longitudinal metagenomic analysis from a naïve state to a treated state for IBD patients is therefore necessary to clarify the association between disease onset and specific bacterial species.

Inflammatory cytokines are crucial to the pathogenesis of IBD [20]. TNF is a central driver of inflammation in mucosal tissues, as evidenced by the anti-inflammatory effects of a neutralizing antibody against TNF that is currently used worldwide for the treatment of both UC and CD $[21,22]$. IL- 6 produced by lamina propria myeloid cells prevents apoptosis of $\mathrm{T}$ cells and activates macrophages [23]. Antibody-mediated blockade of IL-6 signaling resulted in clinically beneficial responses in a subgroup of CD patients in a clinical trial [24]. IL-6 is also necessary for differentiation of Th17 cells. IL-17A produced by Th17 cells in the lamina propria is abundant in the mucosa of both UC and CD patients [20]. Although neutralization of IL-17A was not effective in the relief of colitis in a mouse model [25] or in a clinical study of CD patients [26], another Th17-type cytokine, IL-21, also has pro-inflammatory effects and may be a suitable therapeutic target [20]. IL-23 is mainly produced by antigen-presenting cells to stabilize Th17 cells, but also activates macrophages. A neutralizing antibody against IL-12p40, a subunit of IL-23, is now used for the treatment of CD patients worldwide [27]. Interestingly, in this study, cytokine expression profiles in colon tissues of $I l 10^{-1-}$ mice were dependent on the composition of the transplanted microbiota. The UC microbiota tended to increase the expression of the Il6-Il17a axis, while the $C D$ microbiota increased $I l 23 a$ expression in the colon (Fig. 3a). In addition, cytokine profiles were dependent on the genotypes of the E. faecium strains in the $I l 10^{-1-}$ mice (Fig. 6d). Strain IB51a increased expression of Thf, Il1b, Il12b, and $I l 17 a$ in gnotobiotic conditions, but original IBD51 feces only increased Il23a expression (Fig. 6f). Even in the same host background, differences in the bacterial community composition might affect the specific cytokine expression profiles of the colon tissues. This study also showed distinct differences in bacterial species composition between the UC and CD microbiota and the HD microbiota (Fig. 1a, b), as previously reported [3]. Similar to the current study, 
differences in Klebsiella pneumoniae genotype induced different inflammation profiles in the colon of $1 l 10^{-/-}$ mice [28]. Therefore, accumulating information regarding the relationship between the expression of intestinal cytokines and the composition of the gut microbiota, as well as the genetic background of the host, may help in the development of individualized treatment protocols to appropriately neutralize various cytokines.

Ill1 ${ }^{-/-}$mice, which lack anti-inflammatory cytokine IL-10 and show spontaneous colitis in a gut microbiotadependent manner [12], were used in this study. Because IL10 gene polymorphisms contribute to UC [29] and CD [30] susceptibility in humans, the $I l 10^{-/-}$mouse model is a good approximation of the genetic susceptibility of IBD patients. However, multiple alleles are usually involved in the pathogenesis of human IBD [31], and not all IBD patients have an IL10 polymorphism. Therefore, further investigation using other mouse models of IBD is necessary to validate the results of the current study, although mouse models of genetic susceptibility to colitis are scarce. Microbiota-dependent patterns of cytokine production also need to be confirmed in other mouse models. A limitation of this study was that transplantation of human feces into mice after depletion of native mouse microbiota with antibiotics resulted in incomplete restoration of the human microbiota (Additional file 2: Figure S1). Thus, we were able to study effects of subject-unique microbiota by fecal transplantation, but the microbiota were not the same as the original microbiota of the donor human. Despite this limitation of the mouse experiments in this study, the presence of E. faecium was associated with increased disease extent and the requirement for multiple medications in UC patients with a non-specific genetic background. Taken together, the results of the current study suggest that E. faecium may promote colonic inflammation in UC patients. To validate the inflammatory effects of E. faecium in IBD patients, well-designed clinical studies using a larger UC population are necessary.

Probiotic E. faecium strains NCIMB 11181 [15] and SF68 $[14,32]$ have been used for many years to maintain animal health; however, the detailed molecular mechanisms underlying their probiotic effects have not been fully elucidated. In comparison, the virulence factors of E. faecium have been extensively studied because of the significant health concern caused by vancomycin-resistant $E$. faecium strains in hospitalized patients [33]. Unlike streptococci and staphylococci, most enterococci do not produce proinflammatory toxins; instead, they possess many genes encoding adhesion proteins that mediate adherence to host tissues [33]. Acm is one such adhesion protein produced by clinical E. faecium strains. Acm binds collagen in host tissues, and deletion of $\mathrm{acm}$ results in attenuation of $E$. faecium-mediated endocarditis in an animal model of disease
[34]. Surface protein Esp in E. faecium appears to have been acquired as part of a pathogenicity island, and promotes biofilm formation and urinary tract infection in mice [35]. Adhesion and biofilm formation are important properties involved in the translocation of luminal bacteria in colon tissues [33]. The genetic analysis of $10 \mathrm{E}$. faecium strains in the current study identified that pathways for secondary bile acid biosynthesis were abundant in the proinflammatory cluster of $E$. faecium strains. Because particular types of bile acids control biofilm formation of Enterococcus [36], polarization of host macrophages, generation of oxidative stress [37], and expansion of pathobionts in the colon [38], the ability of "inflammatory" E. faecium to alter the composition of bile acids in the intestinal environment may be a key feature of pathogenesis of E. faecium in colitis. Abundance of the pathway of lipoic acid metabolism was also different between the inflammatory and the probiotic clusters. Lipoic acids are considered as antioxidants and effective to relieve colitis in pre-clinical animal studies [16]. Because E. faecium strains in the inflammatory cluster possessed ability to generate more ROS than the probiotic cluster, ROS-related pathogenic effect may be involved in the colitis induced by inflammatory E. faecium. In several fecal transplantation, E. faecium caused colitis in $\mathrm{Il1O}^{-/-}$mice with low bacterial load (Additional file 2: Figure S5). Ability of inflammatory E. faecium in generating metabolites related to bile acids and ROS might account for a reason why low bacterial load of the strains promoted colitis.

\section{Conclusions}

Metagenomic analysis of the fecal microbiota of IBD patients and transplantation of feces from the corresponding patients into genetically susceptible animals confirmed a causal relationship between inflammatory E. faecium strains and colitis. Because the cytokine expression profile in the colon may partly be dependent on the gut microbiome, characterizing the composition of a patient's gut microbiota may lead to personalized antibody therapy for IBD patients in the future.

\section{Methods \\ Clinical study population}

This study aimed to examine fecal microbiota of IBD patients who visited Kanazawa University Hospital in various stages including both active and remission stages. Between February 2014 and March 2015, 60 IBD patients enrolled in the study. Among them, 55 subjects received colonoscopy to evaluate disease status. To investigate the composition and metagenomes of the gut microbiota of the different patient groups, fecal samples were collected from patients immediately after enrollment and stored at $-80^{\circ} \mathrm{C}$. Among the 55 subjects, $16 \mathrm{UC}$ patients and $8 \mathrm{CD}$ patients provided us with good and sufficient feces 
immediately after enrollment, and these feces were used for the study. Feces that were too watery or that were left at room temperature for $>3 \mathrm{~h}$ were not used. Patients received both written and oral information before consenting to participate in this study. Healthy individuals were first tested to confirm that they met the following inclusion criteria: body mass index $<25 \mathrm{~kg} / \mathrm{m}^{2}$, normal blood pressure, normal serum cholesterol, normal blood glucose and hemoglobin A1c levels, normal serum aspartate transaminase and alanine aminotransferase levels, no anemia, no fatty liver noted by ultrasonography, and no past history of cancer. In total, 13 individuals were enrolled as HD. Immediately after consent, feces were collected from the $\mathrm{HD}$ and stored at $-80^{\circ} \mathrm{C}$. This study was approved by the Ethics Committee of Kanazawa University (approval number 2012-109).

\section{Animals}

Male and female $I l 10^{-1-}$ mice with a C57BL/6j background were purchased from Jackson Laboratories (Bar Harbor, ME, USA). Mice were housed in individually ventilated racks in an animal facility with access to autoclaved food and water ad libitum. Stringent husbandry techniques, including a strictly enforced order of cage handling and scrupulous attention to environmental sanitization, were followed to prevent contamination. Germ-free $I l 10^{-/-}$mice with a C57BL/6j background were generated by Japan CLEA (Tokyo, Japan). Germ-free or gnotobiotic mice were housed in germ-free isolators (ICM, Tsukuba, Japan) in the animal facility of Kanazawa University. All animal procedures were performed in accordance with the Guidelines on the Care and Use of Laboratory Animals issued by Kanazawa University and were approved by the Ethical Committee for Animal Experiments of Kanazawa University (approval number 143267).

\section{Fecal transplantation and bacterial inoculation}

Frozen fecal samples were thawed and resuspended in a 10 times volume $(\mathrm{w} / \mathrm{v})$ of reduced phosphate-buffered saline (PBS) containing $0.1 \%$ resazurin $(\mathrm{w} / \mathrm{v})$ and $0.05 \% \mathrm{~L}-$ cysteine- $\mathrm{HCl}$ (Sigma-Aldrich, St. Louis, MO, USA) under anaerobic conditions $\left(80 \% \mathrm{~N}_{2}, 10 \% \mathrm{H}_{2}, 10 \% \mathrm{CO}_{2}\right)$ in an anaerobic chamber (RUSKINN, Bridgend, UK), before being filtered through a $100-\mu \mathrm{m}$ pore size nylon membrane filter. Depletion of the gut microbiota was performed as previously described [39], with slight modification. Briefly, 8- to 12-week-old C57BL6 mice were treated with antibiotics, including doripenem hydrate $(0.25 \mathrm{~g} / \mathrm{L}$; Shionogi, Japan) and vancomycin hydrochloride $(0.5 \mathrm{~g} / \mathrm{L}$; Shionogi), via water for 2 weeks prior to experimentation to deplete the gut microbiota. Fecal suspensions were then inoculated into the microbiota-depleted mice by oral gavage $(100 \mu \mathrm{L})$ and rectally $(100 \mu \mathrm{L})$ using a flexible plastic tube on days 1 and 2 after cessation of antibiotic treatment. For bacterial inoculation experiments, E. faecium strains ATCC 19434 (ATCC, Manassas, VA, USA), NCIMB 11181 (NCIMB, Aberdeen, UK), SF68 (also known as NCIMB 10415; PURINA, Largo, FL, USA), or human feces-derived strains were prepared at a concentration of $2.5 \times 10^{8}$ colony-forming units $(\mathrm{CFU}) / 100 \mu \mathrm{L}$ in PBS. Suspensions were then inoculated into microbiota-depleted mice as described for the inoculation of fecal suspension. The microbiota-depleted mice were housed individually in separate isolators following inoculation with fecal suspension or specific bacterial strains.

\section{Whole-genome shotgun and 16S rRNA amplicon sequencing analysis of fecal and bacterial DNA extracts} Microbial DNA was extracted from the stored fecal samples using a PowerFecal DNA Isolation Kit according to the manufacturer's instructions (MO BIO, Carlsbad, CA, USA), although 0.1-mm glass beads (MO BIO) rather than 0.7-mm garnet beads were used to homogenize feces.

For whole-genome shotgun sequencing, DNA fragmentation was performed using a Nextera DNA Library Prep Kit (Illumina, San Diego, CA, USA) with incubation at $55^{\circ} \mathrm{C}$ for $5 \mathrm{~min}$. Following product clean-up using a DNA Clean \& Concentrator-5 Kit (ZYMO, Irvine, CA, USA), indexing PCR was performed using a Nextera Index Kit (Illumina), with an initial cycle of $72{ }^{\circ} \mathrm{C}$ for 3 min and $98^{\circ} \mathrm{C}$ for $30 \mathrm{~s}$, followed by 5 cycles of $98^{\circ} \mathrm{C}$ for $10 \mathrm{~s}, 63{ }^{\circ} \mathrm{C}$ for $30 \mathrm{~s}$, and $72{ }^{\circ} \mathrm{C}$ for $3 \mathrm{~min}$. PCR products were purified from oligo DNA contaminants using AMPure XP beads (Beckman Coulter, Fullerton, CA, USA) and then quantified using a 2100 Bioanalyzer and High Sensitivity DNA Kit (Agilent Technologies, Santa Clara, CA, USA). The libraries were pooled, and sequencing was performed using the MiSeq system (Illumina) with a MiSeq Reagent Kit V3 (600 cycles; Illumina).

Preprocessing of the acquired sequences was performed as follows. After trimming of bases with low-quality scores from the ends of the acquired sequences, the sequences were filtered at a Q-score cutoff of 20 using FASTX Toolkit [40]. Paired-end joining was performed using MacQIIME v1.9.1 [41]. After mapping of the resultant sequences against UCSC human reference genome hg19 using bowtie2 version 2.2.4 [42], human genome sequences were removed using SAMtools-1.2 [43]. Finally, PCR duplicates were removed using PRINSEQ version 0.20 .4 [40]. The average number of reads per sample before and after quality control was 1,544,241 $\pm 687,438$ and $1,106,822 \pm 560,821$ (mean \pm SD), respectively.

The resultant FASTA files were used for taxonomic analysis of the fecal samples using MetaPhlAn2 version 2.0.0 [44]. Metabolic pathways indicated by the sequences were identified using HUMAnN2 [45] version 0.1.9. A KEGG version 56 database was generated using DIAMOND version 0.7.5 [46] and used for HUMAnN2 
analysis. After normalization of the abundance to 1 million reads, comparisons between groups were performed by LDA using the linear discriminative analysis effect size (LEfSe) tool [47]. The Bray-Curtis dissimilarities among the gut microbiota communities were calculated based on taxonomic or metabolic pathway data, and the resultant distances were visualized by PCoA. Statistical differences between communities were tested using PERMANOVA with MacQIIME version 1.9.1.

Whole-genome shotgun sequencing of individual $E$. faecium strains was performed as described for fecal metagenome analysis. Assembly of the obtained pairedend FASTQ files was performed using SPAdes version 3.12.0 [48]. The resultant scaffold FASTA files were used to annotate genomic features in PROKKA version 1.12 [49]. Hierarchical clustering of 1683 genes from the 10 E. faecium strains annotated by PROKKA was performed using an average linkage method in Genesis version 1.7.6 [50]. KEGG-based metabolic pathways indicated by the sequences were also identified using HUMAnN2 version 0.1 .9 , and comparisons between "inflammatory" and "probiotic" clusters were performed by LEfSe.

For 16S rRNA gene sequencing analysis, PCR amplicons were prepared using primers targeting the V3-V4 region of the gene, with Illumina adapter sequences:

Forward 5'-TCGTCGGCAGCGTCAGATGTGT ATAAGAGACAGCCTACGGGNGGCWGCAG-3'; Reverse $5^{\prime}$-GTCTCGTGGGCTCGGAGATGTGTATAAG AGACAGGACTACHVGGGTATCTAATCC-3'.

PCR reactions were performed using KAPA HiFi HotStart Ready Mix (KAPA Biosystems, Wilmington, MA, USA) with an initial step at $95^{\circ} \mathrm{C}$ for $3 \mathrm{~min}$, followed by 25 cycles of $95^{\circ} \mathrm{C}$ for $30 \mathrm{~s}, 55^{\circ} \mathrm{C}$ for $30 \mathrm{~s}$, and $72^{\circ} \mathrm{C}$ for 30 s, with a final elongation at $72{ }^{\circ} \mathrm{C}$ for $5 \mathrm{~min}$. Following purification, indexing PCR was performed using a Nextera XT Index Kit (Illumina) with an initial step at $95^{\circ} \mathrm{C}$ for 3 min, followed by 8 cycles of $95^{\circ} \mathrm{C}$ for $30 \mathrm{~s}, 55^{\circ} \mathrm{C}$ for $30 \mathrm{~s}$, and $72{ }^{\circ} \mathrm{C}$ for $30 \mathrm{~s}$, with a final elongation step at $72^{\circ} \mathrm{C}$ for $5 \mathrm{~min}$. Libraries were pooled and mixed with the PhiX Control Library (Illumina) before being sequenced using the MiSeq system with a MiSeq Reagent Kit V3 (600 cycles). Downstream processing of the sequences was performed using MacQIIME version 1.9.1 [41]. After paired-end sequence joining and trimming of low-quality reads, the resulting sequences had lengths $442-464 \mathrm{bp}$. The average number of reads per sample after quality control was 96,311 $\pm 35,047$ (mean \pm SD). Operational taxonomic units (OTUs) were assigned using UCLUST. For OTU analysis, sequences were clustered, and then, those with $>97 \%$ similarity were binned into the same OTU. Taxonomic assignment of representative sequences from each OTU was performed using RDP Classifier using the Greengenes reference database [51] clustered at 97\% identity. Genus-level taxonomy was summarized and used for subsequent analyses. Following the generation of a phylogenetic tree, unweighted UniFrac distances for the gut microbiota communities were calculated, with the resultant distances visualized by PCoA.

All sequencing data and metadata were archived in the NCBI Sequence Read Archive under BioProject numbers PRJNA511372 and 511382.

\section{Real-time PCR}

Total RNA extraction and cDNA synthesis were performed as previously described [52]. Briefly, colon tissue samples were fixed in RNAlater RNA Stabilization Reagent (Qiagen, Hilden, Germany) and stored at $-80^{\circ} \mathrm{C}$ until processing. Total RNA was isolated from the specimens using an RNeasy Mini Kit (Qiagen) according to the manufacturer's instructions. A High Capacity cDNA Archive Kit (Applied Biosystems, Foster City, CA, USA) was used to reverse transcribe $500 \mathrm{ng}$ of total RNA into first-strand cDNA. Quantitative PCR was carried out using a real-time $7900 \mathrm{HT}$ Sequence Detection System (Applied Biosystems). TaqMan probes used for real-time PCR were as follows: Tnf (Mm00443260_g1), Il1b (Mm00434228_m1), Il6 (Mm00446190_m1), Il12b (Mm01288989_m1), Il17a (Mm00439618_m1), and Il23a (Mm00518984_m1) (Applied Biosystems). To control for variations in the amount of DNA available for PCR, target gene expression in each sample was normalized relative to the expression of endogenous control gene $\beta$-actin (Applied Biosystems) using the $\Delta \Delta \mathrm{Ct}$ method. PCR amplification was performed with an initial cycle of $50^{\circ} \mathrm{C}$ for $2 \mathrm{~min}$ and $95^{\circ} \mathrm{C}$ for $10 \mathrm{~min}$, followed by 40 cycles of $95^{\circ} \mathrm{C}$ for 15 s and $60^{\circ} \mathrm{C}$ for $1 \mathrm{~min}$.

Detection of E. faecium and E. faecalis in fecal samples was performed as previously described [53]. Briefly, $2 \mathrm{ng}$ of fecal DNA was amplified with SYBR Green (Qiagen) and primers Efm12 or Efl6 [53] to detect E. faecium or E. faecalis, respectively, by reaction at $94{ }^{\circ} \mathrm{C}$ for $10 \mathrm{~min}$, followed by 40 cycles of $94{ }^{\circ} \mathrm{C}$ for $15 \mathrm{~s}$ and $60^{\circ} \mathrm{C}$ for 1 min. To draw a standard curve, DNA extracted from $E$. faecium strain ATCC 19434 or E. faecalis strain V583 (ATCC) was used, and copy number was calculated.

\section{Histological analysis}

Colon samples were fixed in $10 \%$ buffered formalin using a "Swiss roll" technique [54]. Fixed tissues were embedded in paraffin and stained with hematoxylin and eosin. Hematoxylin and eosin-stained sections were used for histological scoring. Tissue sections were coded to eliminate pathologist bias and scored as previously described [54], with slight modification. Briefly, the proximal colon, distal colon, and rectum from each mouse were scored based on the severity of mucosal epithelial changes, degree of inflammation, and extent of pathology (Additional file 1: Table S7). The segment score 
was calculated by summing the severity scores: [segment score $=$ mucosal score + inflammation score + extent of segment affected in any manner (extent 1$)+$ extent of segment affected at level 3 or 4 in $\mathrm{M}$ or I score (extent $2)$ ], where the maximum segment score was 15 . The total score for each mouse was calculated by summing the scores from the individual segments (maximum total score of 45). Subsequently, the mean total scores were calculated for each group.

\section{Bacterial culture}

E. faecium strains were proliferated in Brain Heart Infusion (BHI) medium (Eiken Chemical Co., Tokyo, Japan). To isolate E. faecium strains from human feces, fecal samples were streaked onto ECS agar plates (Eiken Chemical Co.). Single colonies were picked from the selection agar and cultivated on EF agar plates (Nissui, Japan), upon which E. faecalis forms purple colonies and $E$. faecium produces orange colonies. Individual orange colonies were picked and proliferated in BHI broth. Species identification was carried out using PCR-based methods as previously described [53, 55]. Finally, Enterococcus species were confirmed by whole-genome shotgun sequencing as described in the "Whole-genome shotgun and 16S rRNA amplicon sequencing analysis of fecal and bacterial DNA extracts"section. Fecal suspension was cultured on EF agar plates to obtain CFU counts, and colonies were confirmed as E. faecium by PCR-based methods.

\section{Intracellular cytokine staining by flow cytometry}

Single cell suspension was obtained from the colonic lamina propria. The colon tissues were cut into $1-\mathrm{cm}$ pieces and incubated with $5 \mathrm{mM}$ ethylenediaminetetraacetic acid (Invitrogen) in Hanks' balanced salt solution at $37^{\circ} \mathrm{C}$ for $20 \mathrm{~min}$, followed by washing with HBSS three times. Next, the tissue was minced with scissors and transferred into conical tubes containing $10 \mathrm{~mL}$ RPMI 1640 medium (Thermo Fisher, Waltham, MA, USA) and $0.5 \mathrm{mg} / \mathrm{mL}$ collagenase (Sigma-Aldrich, St. Louis, MO, USA) and incubated at $150 \mathrm{rpm}$ and $37{ }^{\circ} \mathrm{C}$ for $30 \mathrm{~min}$. The single cell suspension was collected and passed through $100-\mu \mathrm{m}$ and $40-\mu \mathrm{m}$ cell strainers and washed twice with RPMI 1640. For analysis of IL-17A expression, the single colonic lamina propria cells were restimulated in complete RPMI 1640 with $5 \mathrm{ng} / \mathrm{mL}$ phorbol 12-myristate 13 -acetate and $500 \mathrm{ng} / \mathrm{mL}$ ionomycin in the presence of $2 \mu \mathrm{M}$ monensin (Sigma-Aldrich) for $3 \mathrm{~h}$ at $37^{\circ} \mathrm{C}$. For analysis of IL- 6 and TNF expression, the cells were incubated in complete RPMI 1640 in the presence of $2 \mu \mathrm{M}$ monensin for $3 \mathrm{~h}$ at $37^{\circ} \mathrm{C}$. Dead cells were excluded from all analyses using a LIVE/DEAD Fixable Aqua Dead Cell Stain Kit (Thermo Fisher). For intracellular cytokine staining, cells were fixed with IC
Fixation Buffer (Thermo Fisher). Fluorescent dyeconjugated antibodies were used to stain CD45, CD3 (BD Biosciences, Franklin Lakes, NJ, USA), CD4, CD11c, MHC-class II, IL-17, TNF, and IL-6 (BioLegend, San Diego, CA, USA). All data were acquired on a FACS Aria II flow cytometer (BD Biosciences) and analyzed using FlowJo V.10 software (FlowJo, Ashland, OR, USA).

\section{BSH assay}

E. faecium strains were tested for hydrolase activity against glyco-conjugated bile acid as previously described [56]. Overnight MRS broth cultures were streaked on MRS agar supplemented with $2 \mathrm{mM}$ GDCA (Sigma; G3258). The plate was then incubated for $48 \mathrm{~h}$ at $37^{\circ} \mathrm{C}$. BSH activity was detectable when deoxycholic acid precipitated in the agar medium around a colony.

\section{ROS measurement in culture supernatant}

OxiSelect In vitro ROS/RNS assay kit (green fluorescence) (Cell Biolabs, cat. STA-347) was used to measure the ROS/RNS (reactive nitrogen species) in culture supernatant as previously described [57] with modification. After E. faecium strains were cultured in BHI medium overnight, $50 \mu \mathrm{L}$ of the supernatants were mixed with $50 \mu \mathrm{L}$ of catalyst (provided in the kit) to accelerate the oxidative reaction. Following $5 \mathrm{~min}$ incubation at room temperature, $100 \mu \mathrm{L}$ of $\mathrm{DCFH}-\mathrm{DiOxyQ}$ probe solution was added to the mixture to measure the total free radical population. DCFH probe can react with free radical molecules that are representative of both ROS and RNS. The samples were incubated at room temperature for $30 \mathrm{~min}$ and read with a fluorescence plate reader at $\mathrm{Ex} / \mathrm{Em}=480 /$ $530 \mathrm{~nm}$. The standard curve of $\mathrm{H}_{2} \mathrm{O}_{2}$ was used to semiquantify the free radical content in the culture supernatant samples. Then, relative ROS level was determined.

\section{Statistical analyses}

Statistical analyses of taxonomic and KEGG pathway comparisons between microbiota communities were performed using the LEfSe tool [47]. Statistical differences between two values were analyzed using a Mann-Whitney $U$ test or the Kruskal-Wallis test followed by Dunn's test for correction of multiple comparisons in GraphPad Prism 7 (GraphPad Software, San Diego, CA, USA). Statistical differences between treatments or in the percentages of disease extent were analyzed by $\chi^{2}$ test in GraphPad Prism 7. A $P$ value $<0.05$ was considered statistically significant. Linear regression coefficients between pathology scores, percentage body weight changes, and Tnf expression levels were calculated using GraphPad Prism 7. Spearman's rank correlation was analyzed using GraphPad Prism 7. 


\section{Supplementary information}

Supplementary information accompanies this paper at https://doi.org/10. 1186/s13059-019-1879-9.

Additional file 1: Table S1. Baseline demographic characteristics of the subjects. Table S2. Disease characteristics of the UC patients. Table S3. Disease characteristics of the CD patients. Table S4. Comparison of KEGG pathway abundance in fecal microbiota between HD and UC subjects by linear. Table S5. Comparison of KEGG pathway abundance in fecal microbiota between HD and CD subjects by linear discriminative analysis. Table S6. Correlation between abundance of fecal Enterococcus and pathology/cytokine production. Table S7. Scoring system for histological evaluation of mouse colon tissues.

Additional file 2: Figure S1. $\beta$-Diversity analysis of the bacterial community in mouse feces following fecal transplantation from human subjects. Figure S2. Pathological analysis of colorectal segments of $1 / 10^{-1-}$ mice following fecal transplantation. Figure S3. Cytokine expression of colon in individual groups of mice. Figure S4. Quantitation of E. faecium in mice by culture after fecal transplantation. Figure S5. Quantitation of E. faecium and E. faecalis in mice by PCR after fecal transplantation.

Figure S6. Quantitation of E. faecium in mice by culture after E. faecium inoculation. Figure S7. Effect of inoculation with UC-derived or probiotic E. faecium strain on colitis. Figure S8. Intracellular cytokine staining of colon lamina propria cell after inoculation of E. faecium strain IB51a.

Additional file 3: Figure S9. Hierarchical clustering analysis of the $10 \mathrm{E}$. faecium strains based on 1683 identified genes.

Additional file 4: Figure S10. Bile salt hydrolase (BSH) activity and reactive oxygen species (ROS)-generating ability in E. faecium strains. Figure S11. Presence of E. faecium in feces of UC patients on treatment is not associated with disease activity.

Additional file 5:. Review history.

\section{Acknowledgements}

The authors thank Dr. Souichiro Sasaki (Division of Molecular Bioregulation, Cancer Research Institute, Kanazawa University) for advice regarding animal experiments, Ms. Akiko Katayama (Kanazawa University) for assistance with histological analysis, Ms. Maki Wakabayashi (Kanazawa University) for animal care, and Ms. Masami Kitaoka (Kanazawa University) for collecting fecal samples. We also thank Tamsin Sheen, PhD, from Edanz Group (www edanzediting.com/ac) for editing a draft of this manuscript.

\section{Review history}

The review history is available as Additional file 5.

\section{Peer review information}

Kevin Pang was the primary editor on this article and managed its editorial process and peer review in collaboration with the rest of the editorial team.

\section{Authors' contributions}

JS and NI contributed equally to this work. NI and EM conceived and designed this study. JS and NI acquired, analyzed, and interpreted the data from animal experiments. NI, KK, TK, and YSh collected the human samples. JS, NI, ZW, MY, and YF acquired and analyzed the bacterial experiment data. $\mathrm{NI}$ acquired, analyzed, and interpreted the data from sequencing experiments. AS analyzed the flow cytometry data. JS and NI drafted the manuscript. TarY, YSa, MH, TatY, EM, and SK critically revised the manuscript for important intellectual content. EM and SK supervised this study. All authors read and approved the final manuscript.

\section{Funding}

Not applicable.

\section{Availability of data and materials}

Sequencing data and metadata were archived in the NCBI Sequence Read Archive under BioProject numbers PRJNA511372 [58] and PRJNA511382 [59].

\section{Ethics approval and consent to participate}

The clinical study was approved by the Ethics Committee of Kanazawa University (approval number 2012-109). All animal experiments were performed in accordance with the Guidelines on the Care and Use of Laboratory Animals issued by Kanazawa University and were approved by the Ethical Committee for Animal Experiments of Kanazawa University (approval number 143267). All experimental methods were in accordance with Helsinki Declaration.

\section{Consent for publication}

All participants provided written informed consent for publication of study results of the collected biomaterials paired with anonymized information on clinical data.

\section{Competing interests}

The authors declare that they have no competing interests.

\section{Author details}

'Department of Gastroenterology, Graduate School of Medical Sciences, Kanazawa University, Kanazawa, Ishikawa 920-8641, Japan. ²Department of Bacteriology, Graduate School of Medicinal Sciences, Kanazawa University, Kanazawa, Ishikawa, Japan. ${ }^{3}$ Department of Advanced Medical Technology, Graduate School of Health Medicine, Kanazawa University, Kanazawa, Ishikawa, Japan.

Received: 21 February 2019 Accepted: 4 November 2019

Published online: 25 November 2019

References

1. Honda K, Littman DR. The microbiota in adaptive immune homeostasis and disease. Nature. 2016:535:75-84.

2. Ni J, Wu GD, Albenberg L, Tomov VT. Gut microbiota and IBD: causation or correlation? Nat Rev Gastroenterol Hepatol. 2017;14:573-84.

3. Morgan XC, Tickle TL, Sokol H, Gevers D, Devaney KL, Ward DV, Reyes JA, Shah SA, LeLeiko N, Snapper SB, et al. Dysfunction of the intestinal microbiome in inflammatory bowel disease and treatment. Genome Biol. 2012;13:R79

4. Gevers D, Kugathasan S, Denson LA, Vazquez-Baeza Y, Van Treuren W, Ren B, Schwager E, Knights D, Song SJ, Yassour M, et al. The treatment-naive microbiome in new-onset Crohn's disease. Cell Host Microbe. 2014;15:382-92.

5. Lewis JD, Chen EZ, Baldassano RN, Otley AR, Griffiths AM, Lee D, Bittinger K, Bailey A, Friedman ES, Hoffmann C, et al. Inflammation, antibiotics, and diet as environmental stressors of the gut microbiome in pediatric Crohn's disease. Cell Host Microbe. 2017;22:247.

6. Frank DN, St Amand AL, Feldman RA, Boedeker EC, Harpaz N, Pace NR. Molecular-phylogenetic characterization of microbial community imbalances in human inflammatory bowel diseases. Proc Natl Acad Sci U S A. 2007;104:13780-5

7. Machiels K, Joossens M, Sabino J, De Preter V, Arijs I, Eeckhaut V, Ballet V, Claes K, Van Immerseel F, Verbeke K, et al. A decrease of the butyrateproducing species Roseburia hominis and Faecalibacterium prausnitzii defines dysbiosis in patients with ulcerative colitis. Gut. 2014;63:1275-83.

8. Sokol H, Pigneur B, Watterlot L, Lakhdari O, Bermudez-Humaran LG, Gratadoux JJ, Blugeon S, Bridonneau C, Furet JP, Corthier G, et al. Faecalibacterium prausnitzii is an anti-inflammatory commensal bacterium identified by gut microbiota analysis of Crohn disease patients. Proc Natl Acad Sci U S A. 2008;105:16731-6.

9. Mazmanian SK, Round JL, Kasper DL. A microbial symbiosis factor prevents intestinal inflammatory disease. Nature. 2008;453:620-5.

10. Atarashi K, Tanoue T, Oshima K, Suda W, Nagano Y, Nishikawa H, Fukuda S, Saito T, Narushima S, Hase K, et al. Treg induction by a rationally selected mixture of Clostridia strains from the human microbiota. Nature. 2013:500:232-6.

11. Kim SC, Tonkonogy SL, Albright CA, Tsang J, Balish EJ, Braun J, Huycke MM, Sartor RB. Variable phenotypes of enterocolitis in interleukin 10-deficient mice monoassociated with two different commensal bacteria. Gastroenterology. 2005;128:891-906.

12. Balish $\mathrm{E}$, Warner $\mathrm{T}$. Enterococcus faecalis induces inflammatory bowel disease in interleukin-10 knockout mice. Am J Pathol. 2002;160:2253-7.

13. Steck N, Hoffmann M, Sava IG, Kim SC, Hahne H, Tonkonogy SL, Mair K, Krueger D, Pruteanu M, Shanahan F, et al. Enterococcus faecalis metalloprotease compromises epithelial barrier and contributes to intestinal inflammation. Gastroenterology. 2011;141:959-71.

14. Bednorz C, Guenther S, Oelgeschlager K, Kinnemann B, Pieper R, Hartmann S, Tedin K, Semmler T, Neumann K, Schierack P, et al. Feeding the probiotic 
Enterococcus faecium strain NCIMB 10415 to piglets specifically reduces the number of Escherichia coli pathotypes that adhere to the gut mucosa. Appl Environ Microbiol. 2013;79:7896-904.

15. Chae JP, Pajarillo EA, Oh JK, Kim H, Kang DK. Revealing the combined effects of lactulose and probiotic enterococci on the swine faecal microbiota using 454 pyrosequencing. Microb Biotechnol. 2016;9:486-95.

16. Moeinian M, Abdolghaffari AH, Nilfar S, Momtaz S, Abdollahi M. Effects of alpha lipoic acid and its derivative "andrographolid-lipoic acid-1" on ulcerative colitis: a systematic review with meta-analysis of animal studies. J Cell Biochem. 2019;120:4766-82.

17. Fite A, Macfarlane S, Furrie E, Bahrami B, Cummings JH, Steinke DT, Macfarlane GT. Longitudinal analyses of gut mucosal microbiotas in ulcerative colitis in relation to patient age and disease severity and duration. J Clin Microbiol. 2013;51:849-56.

18. Sha S, Xu B, Wang X, Zhang Y, Wang H, Kong X, Zhu H, Wu K. The biodiversity and composition of the dominant fecal microbiota in patients with inflammatory bowel disease. Diagn Microbiol Infect Dis. 2013;75:245-51.

19. Neut C, Bulois P, Desreumaux P, Membre JM, Lederman E, Gambiez L, Cortot A, Quandalle P, van Kruiningen H, Colombel JF. Changes in the bacterial flora of the neoterminal ileum after ileocolonic resection for Crohn's disease. Am J Gastroenterol. 2002;97:939-46.

20. Neurath MF. Cytokines in inflammatory bowel disease. Nat Rev Immunol. 2014;14:329-42.

21. Danese S, Fiocchi C. Ulcerative colitis. N Engl J Med. 2011;365:1713-25.

22. Baumgart DC, Sandborn WJ. Crohn's disease. Lancet. 2012;380:1590-605.

23. Atreya R, Mudter J, Finotto S, Mullberg J, Jostock T, Wirtz S, Schutz M, Bartsch B, Holtmann M, Becker C, et al. Blockade of interleukin 6 trans signaling suppresses T-cell resistance against apoptosis in chronic intestinal inflammation: evidence in crohn disease and experimental colitis in vivo. Nat Med. 2000;6:583-8.

24. Ito H, Takazoe M, Fukuda Y, Hibi T, Kusugami K, Andoh A, Matsumoto T, Yamamura T, Azuma J, Nishimoto N, et al. A pilot randomized trial of a human anti-interleukin-6 receptor monoclonal antibody in active Crohn's disease. Gastroenterology. 2004;126:989-96 discussion 947.

25. O'Connor W Jr, Kamanaka M, Booth CJ, Town T, Nakae S, Iwakura Y, Kolls JK, Flavell RA. A protective function for interleukin 17A in T cell-mediated intestinal inflammation. Nat Immunol. 2009;10:603-9.

26. Hueber W, Sands BE, Lewitzky S, Vandemeulebroecke M, Reinisch W Higgins PD, Wehkamp J, Feagan BG, Yao MD, Karczewski M, et al. Secukinumab, a human anti-IL-17A monoclonal antibody, for moderate to severe Crohn's disease: unexpected results of a randomised, double-blind placebo-controlled trial. Gut. 2012;61:1693-700.

27. Feagan BG, Sandborn WJ, Gasink C, Jacobstein D, Lang Y, Friedman JR, Blank MA, Johanns J, Gao LL, Miao Y, et al. Ustekinumab as induction and maintenance therapy for Crohn's disease. N Engl J Med. 2016;375:1946-60.

28. Atarashi K, Suda W, Luo C, Kawaguchi T, Motoo I, Narushima S, Kiguchi Y, Yasuma $\mathrm{K}$, Watanabe $\mathrm{E}$, Tanoue $\mathrm{T}$, et al. Ectopic colonization of oral bacteria in the intestine drives TH1 cell induction and inflammation. Science. 2017;358:359-65.

29. Franke A, Balschun T, Karlsen TH, Sventoraityte J, Nikolaus S, Mayr G, Domingues FS, Albrecht M, Nothnagel M, Ellinghaus D, et al. Sequence variants in IL10, ARPC2 and multiple other loci contribute to ulcerative colitis susceptibility. Nat Genet. 2008;40:1319-23.

30. Franke A, McGovern DP, Barrett JC, Wang K, Radford-Smith GL, Ahmad T, Lees CW, Balschun T, Lee J, Roberts R, et al. Genome-wide meta-analysis increases to 71 the number of confirmed Crohn's disease susceptibility loci. Nat Genet. 2010:42:1118-25.

31. Khor B, Gardet A, Xavier RJ. Genetics and pathogenesis of inflammatory bowel disease. Nature. 2011;474:307-17.

32. Benyacoub J, Czarnecki-Maulden GL, Cavadini C, Sauthier T, Anderson RE, Schiffrin E, von der Weid T. Supplementation of food with Enterococcus faecium (SF68) stimulates immune functions in young dogs. J Nutr. 2003;133:1158-62.

33. Arias CA, Murray BE. The rise of the Enterococcus: beyond vancomycin resistance. Nat Rev Microbiol. 2012;10:266-78.

34. Nallapareddy SR, Singh KV, Okhuysen PC, Murray BE. A functional collagen adhesin gene, acm, in clinical isolates of Enterococcus faecium correlates with the recent success of this emerging nosocomial pathogen. Infect Immun. 2008;76:4110-9.

35. Leendertse M, Heikens E, Wijnands LM, van Luit-Asbroek M, Teske GJ, Roelofs JJ, Bonten MJ, van der Poll T, Willems RJ. Enterococcal surface protein transiently aggravates Enterococcus faecium-induced urinary tract infection in mice. J Infect Dis. 2009;200:1162-5.

36. McKenney PT, Yan J, Vaubourgeix J, Becattini S, Lampen N, Motzer A, Larson PJ, Dannaoui D, Fujisawa S, Xavier JB, Pamer EG. Intestinal bile acids induce a morphotype switch in vancomycin-resistant Enterococcus that facilitates intestinal colonization. Cell Host Microbe. 2019;25:695-705 e695.
37. Jia W, Xie G, Jia W. Bile acid-microbiota crosstalk in gastrointestinal inflammation and carcinogenesis. Nat Rev Gastroenterol Hepatol. 2018;15:111-28.

38. Devkota S, Wang Y, Musch MW, Leone V, Fehlner-Peach H, Nadimpalli A, Antonopoulos DA, Jabri B, Chang EB. Dietary-fat-induced taurocholic acid promotes pathobiont expansion and colitis in II10-/- mice. Nature. 2012;487:104-8.

39. lida N, Dzutsev A, Stewart CA, Smith L, Bouladoux N, Weingarten RA, Molina DA, Salcedo R, Back T, Cramer S, et al. Commensal bacteria control cancer response to therapy by modulating the tumor microenvironment. Science. 2013;342:967-70.

40. Schmieder R, Edwards R. Quality control and preprocessing of metagenomic datasets. Bioinformatics. 2011;27:863-4.

41. Caporaso JG, Kuczynski J, Stombaugh J, Bittinger K, Bushman FD, Costello EK, Fierer N, Pena AG, Goodrich JK, Gordon Jl, et al. QIIME allows analysis of high-throughput community sequencing data. Nat Methods. 2010;7:335-6.

42. Langmead B, Salzberg SL. Fast gapped-read alignment with Bowtie 2. Nat Methods. 2012:9:357-9.

43. Li H, Handsaker B, Wysoker A, Fennell T, Ruan J, Homer N, Marth G, Abecasis G, Durbin R, Genome Project Data Processing S. The sequence alignment/ map format and SAMtools. Bioinformatics. 2009;25:2078-9.

44. Segata N, Waldron L, Ballarini A, Narasimhan V, Jousson O, Huttenhower C. Metagenomic microbial community profiling using unique clade-specific marker genes. Nat Methods. 2012;9:811-4.

45. Abubucker S, Segata N, Goll J, Schubert AM, Izard J, Cantarel BL, RodriguezMueller B, Zucker J, Thiagarajan M, Henrissat B, et al. Metabolic reconstruction for metagenomic data and its application to the human microbiome. PLoS Comput Biol. 2012;8:e1002358.

46. Buchfink B, Xie $\mathrm{C}$, Huson DH. Fast and sensitive protein alignment using DIAMOND. Nat Methods. 2015;12:59-60.

47. Segata N, lzard J, Waldron L, Gevers D, Miropolsky L, Garrett WS, Huttenhower C. Metagenomic biomarker discovery and explanation. Genome Biol. 2011;12:R60.

48. Bankevich A, Nurk S, Antipov D, Gurevich AA, Dvorkin M, Kulikov AS, Lesin VM, Nikolenko SI, Pham S, Prjibelski AD, et al. SPAdes: a new genome assembly algorithm and its applications to single-cell sequencing. J Comput Biol. 2012;19:455-77.

49. Seemann T. Prokka: rapid prokaryotic genome annotation. Bioinformatics. 2014;30:2068-9.

50. Sturn A, Quackenbush J, Trajanoski Z. Genesis: cluster analysis of microarray data. Bioinformatics. 2002:18:207-8.

51. DeSantis TZ, Hugenholtz P, Larsen N, Rojas M, Brodie EL, Keller K, Huber T, Dalevi D, Hu P, Andersen GL. Greengenes, a chimera-checked 16S rRNA gene database and workbench compatible with ARB. Appl Environ Microbiol. 2006;72:5069-72.

52. lida N, Nakamoto Y, Baba T, Nakagawa H, Mizukoshi E, Naito M, Mukaida N, Kaneko S. Antitumor effect after radiofrequency ablation of murine hepatoma is augmented by an active variant of CC Chemokine ligand 3/ macrophage inflammatory protein-1alpha. Cancer Res. 2010;70:6556-65.

53. Nagamine K, Hung GC, Li B, Lo SC. DNA sequence signatures for rapid detection of six target bacterial pathogens using PCR assays. Microbiol Insights. 2015;8:7-14.

54. Burich A, Hershberg R, Waggie K, Zeng W, Brabb T, Westrich G, Viney JL, MaggioPrice L. Helicobacter-induced inflammatory bowel disease in IL-10- and T celldeficient mice. Am J Physiol Gastrointest Liver Physiol. 2001;281:G764-78.

55. Ryu H, Henson M, Elk M, Toledo-Hernandez C, Griffith J, Blackwood D, Noble R, Gourmelon M, Glassmeyer S, Santo Domingo JW. Development of quantitative PCR assays targeting the 165 rRNA genes of Enterococcus spp. and their application to the identification of enterococcus species in environmental samples. Appl Environ Microbiol. 2013;79:196-204.

56. Fang F, Li Y, Bumann M, Raftis EJ, Casey PG, Cooney JC, Walsh MA, O'Toole PW. Allelic variation of bile salt hydrolase genes in Lactobacillus salivarius does not determine bile resistance levels. J Bacteriol. 2009;191:5743-57.

57. Wang H, Nicolay BN, Chick JM, Gao X, Geng Y, Ren H, Gao H, Yang G, Williams JA, Suski JM, et al. The metabolic function of cyclin D3-CDK6 kinase in cancer cell survival. Nature. 2017;546:426-30.

58. Iida N. IBD_WGS; WGS data of fecal microbiota of IBD patients and healthy individuals. NCBI Sequence Read Archive. 2018 https://www.ncbi.nlm.nih. gov/bioproject/PRJNA511372/. Accessed 21 Dec 2018.

59. lida N. IBD_mouse_16SrRNA; 16SrRNA amplicon sequencing of fecal DNA of IBD mouse model. NCBI Sequence Read Archive. 2018 https://www.ncbi. nlm.nih.gov/bioproject/PRJNA511382/. Accessed 21 Dec 2018.

\section{Publisher's Note}

Springer Nature remains neutral with regard to jurisdictional claims in published maps and institutional affiliations. 